\title{
Wireless Hemodynamic Monitoring in Patients with Heart Failure
}

\author{
Matthew M. Lander ${ }^{1}$ (D) Nael Aldweib ${ }^{1} \cdot$ William T. Abraham ${ }^{2}$ \\ Accepted: 17 December 2020 / Published online: 9 January 2021 \\ (C) The Author(s), under exclusive licence to Springer Science+Business Media, LLC part of Springer Nature 2021
}

\begin{abstract}
Purpose of Review Wireless hemodynamic monitoring in heart failure patients allows for volume assessment without the need for physical exam. Data obtained from these devices is used to assist patient management and avoid heart failure hospitalizations. In this review, we outline the various devices, mechanisms they utilize, and effects on heart failure patients.

Recent Findings New applications of these devices to specific populations may expand the pool of patients that may benefit. In the COVID-19 pandemic with a growing emphasis on virtual visits, remote monitoring can add vital ancillary data.

Summary Wireless hemodynamic monitoring with a pulmonary artery pressure sensor is a highly effective and safe method to assess for worsening intracardiac pressures that may predict heart failure events, giving lead time that is valuable to keep patients optimized. Implantation of this device has been found to improve outcomes in heart failure patients regardless of preserved or reduced ejection fraction.
\end{abstract}

Keywords Hemodynamic $\cdot$ CardioMEMs $\cdot$ Heart failure $\cdot$ Impedance

\section{Introduction}

Heart failure (HF) is one of the most common reasons for hospitalization in the USA [1]. Various population-based studies have shown improvements in HF mortality from the proliferation of guideline-directed medical therapies. The prevalence, however, is still high with an estimated 6.5 million Americans living with heart failure, and 26 million people worldwide [2]. By 2030, it is predicted there will be greater than 8 million Americans with heart failure, either with preserved (HFpEF) or reduced ejection fraction (HFrEF) [3]. A heart failure hospitalization (HFH) remains a frequent sentinel event that acts as a marker for future poor outcomes, with a $29.5 \% 1$-year case fatality rate [4]. In a study of over $1000 \mathrm{HF}$

This article is part of the Topical Collection on Nonpharmacologic Therapy: Surgery, Ventricular Assist Devices, Biventricular Pacing, and Exercise

Matthew M. Lander

matthew.lander@ahn.org

1 Cardiovascular Institute at Allegheny Health Network, Pittsburgh, PA, USA

2 Division of Cardiovascular Medicine, The Ohio State University, Columbus, OH, USA patients in Minnesota for a mean follow-up of 4.7 years, $83 \%$ of patients were hospitalized once and $43 \%$ were hospitalized four times [5]. Yet, another study has shown that those that have already had a HFH are hospitalized at greater rates for subsequent heart failure episodes [6]. Highlighting this, from 2013 to 2016, the 1-month readmission rate from the Center for Medicare and Medicaid Services data was $21.6 \%$ for patients with HF [7]. HFH has continually been shown to be a concerning indication of disease progression and future morbidity and mortality.

The health care costs attributable to HF are overwhelming. Projected total costs related to heart failure in 2030 are $\$ 69.7$ billion [3]. HFHs are often long, with a median length of stay of 4 days [8]. The breakdown of costs related to all of cardiovascular disease, including HF, shows that a disproportionate amount is due to hospitalization, as opposed to other care delivery settings [9]. HFHs can therefore be defined as indicators of future morbidity and mortality that are an enormous economic burden. Mitigation of these hospitalizations in turn may improve outcomes, quality of life, and keep healthcare costs down.

However, is it possible to intervene on the natural progression of heart failure and prevent HFH? Diuretics remain a cornerstone of therapy for HF. Guideline-directed medical therapies in HFrEF provide an array of beneficial effects, but 
broad implementation at target doses remains elusive as shown by the Change the Management of Patients with Heart Failure (CHAMP-HF registry) [10]. While intuitive, fluid and sodium restrictions have not been found to improve HF mortality or hospitalizations as expected [11]. A wide variety of devices have been trialed to assess their ability to predict clinical worsening in heart failure and predict HFH. The purpose of this review is to detail the history of HF monitoring devices, describe current and emerging technologies in remote hemodynamic assessment, and highlight the important clinical trials in this field.

\section{Remote Monitoring}

Remote monitoring (RM) offers a unique opportunity to utilize a variety of technologies to maintain a connection with HF patients. Early initiatives focused on extra (remote) education, nursing driven initiatives with telephone support, or telemonitoring systems. These studies attempted to establish a trend of various patient specific data such as heart rate, blood pressure, weights, even self-assessment scores. However, the majority of remote monitoring HF trials have been negative. TELE-HF enrolled 1653 patients at 33 sites with recent HFH and randomized to usual care or an automated telephonebased monitoring system [12]. The primary end point was readmission or death at 180 days. There was no difference in the groups, although there was significant center-to-center variability in adherence to the phone schedule. Several other studies, such as CHAT, PHARM, and DIAL, used telephone support and also saw negative results [13-15].

Integrated systems to make data collection more automated were hypothesized to be an improvement upon simple phone support. By using devices with internet access, noninvasive data can be collected more reliably. But even with this extra degree of monitoring, results have been mixed. The two largest studies are TIM-HF2 and BEAT-HF. Both had over 1400 patients, took patients with recent $\mathrm{HFH}$ and used a telemonitoring system to collect physiologic data, including weight, blood pressure, and heart rate, amongst others. TIMHF2 study showed a reduction in the primary endpoint of proportion of days lost from unplanned cardiovascular hospital admission or all-cause mortality $(4.88 \% 95 \%$ CI $4.55-$ 5.23) [16]. Meanwhile, BEAT-HF showed no difference in the primary endpoint of 180-day readmission after HFH, as well as no difference in mortality [17]. There were differences in the patient population and even the manner in which data was collected and utilized, highlighting the challenges of designing a study that can accurately assess the impact of these management strategies. Furthermore, the reproducibility of the TIM-HF2 intervention, employing a centralized hub and decentralized spoke approach, to geographies outside of that studied in Germany has been questioned.

\section{Continuous Autonomic and Rhythm Assessment with Cardiac Implantable Electronic Devices}

HF can stimulate sympathetic activation and parasympathetic withdrawal arising from decreased cardiac output; hence, heart rate variability can provide insight into HF status [18]. The most common cardiac implantable electronic devices (CIEDs) are implantable cardioverter-defibrillators (ICDs) and cardiac resynchronization therapy defibrillators (CRTDs). These devices can record multiple physiologic variables including tachyarrhythmia burden, heart rate variability, percentage of biventricular pacing, patient activity, abnormal intracardiac electrocardiogram, and intrathoracic impedance (to be discussed more in the following section). Each of these variables is a physiologic marker that is a reflection of autonomic function and can provide prognostic information about cardiac adverse events and function as a precursor for poor clinical outcome and HF exacerbation [19].

A study by Adamson et al. in 2004 used heart rate variability from a CRT-D as a surrogate marker for autonomic activation and predicted cardiovascular death and HFH [20]. The device can assess long-term heart rate variability using a measuring technique called standard deviation of a 5-min median atrial-atrial sensed interval (SDAAM). Absolute SDAAM values are persistently lower in patients at higher risk of mortality and HFH. The change in SDAAM is apparent 3 weeks prior to HF decompensation, meaning that continuous SDAAM measurement can serve as an early warning of clinical decompensation [20]. Unfortunately, while predictive of future events, such measured parameters have not proven to be actionable in a manner that would keep patients well and out of the hospital.

\section{Intrathoracic Impedance Monitoring with CIEDs}

Electrical impedance is a circuit's resistance to current for a given voltage. The clinical application of electrical impedance in HF monitoring utilizes the change in conductance when a small and harmless alternating electrical current is passed across the lungs. Compared to the high resistivity of thoracic tissue (200-5000 $\Omega$ ), blood and fluid (65-150 $\Omega$ ) provide much lower resistances to current. Thus, regions of the body with higher blood or fluid content have lower impedance, whereas regions with more solid tissue will show higher impedance. [21]

Since the 1960s, the principles of impedance have been applied to better understand the presence and degree of thoracic cavity volume [22]. Initial efforts focused on externally measured trans-thoracic impedance via a chest wall electrode system to estimate intrathoracic fluid volume [23]. This 
application is however limited given the impracticality of using many external electrodes to obtain data and the constraints of the electrical properties of the skin $[24,25]$.

The location of a CIED in the thoracic cavity provides a unique opportunity to capture this information. Intrathoracic impedance was found to correlate inversely with left ventricular end-diastolic pressure (LVEDP), pulmonary capillary wedge pressure and fluid balance [26, 27]. A feasibility study by Wang et al. in 2005 found that chronic measurement of intrathoracic impedance with an implantable system effectively revealed changes in thoracic congestion that correlated with directly measured LVEDP [27]. This was one of the first examinations of using an implanted device to apply the principles of impedance to estimate changes in volume status. (Fig. 1).

Once correlation with intracardiac hemodynamics was validated, impedance was tested to assess its ability to predict HFH. Several studies have evaluated the ability of CIEDobtained intrathoracic impedance to predict HF events. The PARTNERS-HF trial was a prospective multicenter observational trial that used multiple parameters, including impedance, to create a monthly score that predicts HFH [28]. Specifically, impedance in this study using Medtronic CRT devices was displayed as a fluid index called Optivol. This index was determined using a mathematical algorithm that calculated the difference between the directly measured impedance and a reference impedance and expressed as ohm-day ( $\Omega$-day). (Fig. 2) A positive total score, called the Cardiac Compass diagnostic report, had a 5.5 fold risk of $\mathrm{HFH}$ in

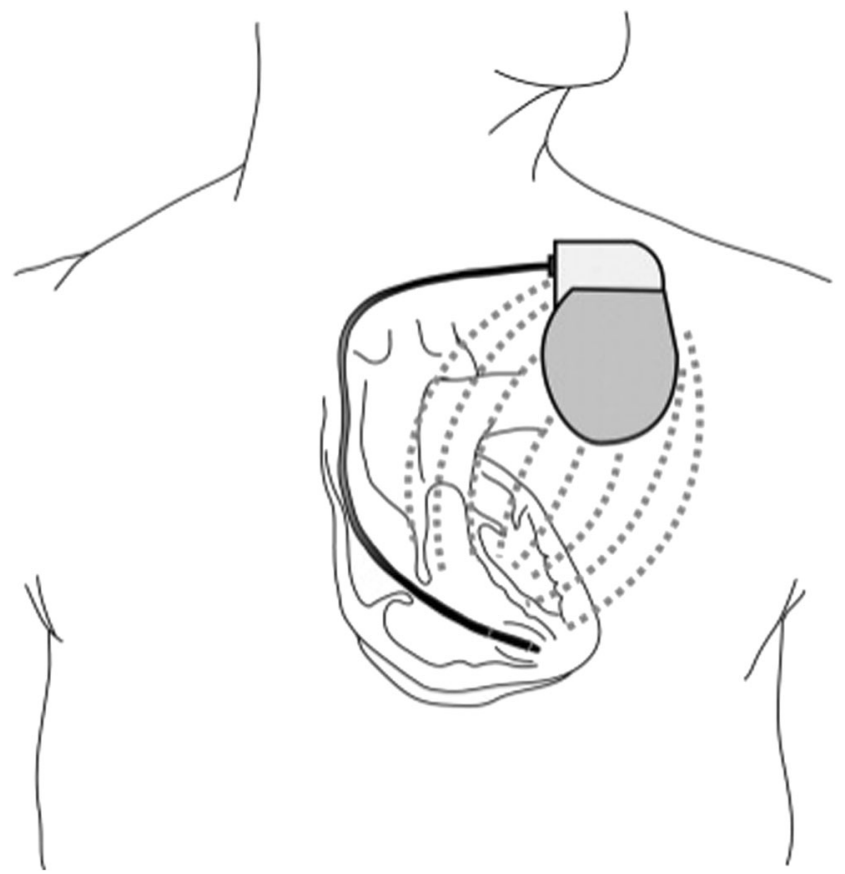

Fig. 1 Schematic illustration of the implanted electrode technique to measure intrathoracic impedance. With permission from Elsevier, original figure by Tang et al. Am Heart J. 2009 March; 157(3): 402-411
1 month. Similarly, the FAST trial compared impedance measurements with weights, and found that impedance had superior sensitivity to predicting episodes of worsening HF [29]. More recently, other trials have shown efficacy of composite scores using a variety of device-obtained physiologic data to predict HFH or clinical worsening, such as Triage-HF and MultiSENSE Study [30, 31].

As seen with telemonitoring, it is challenging to design a clinical trial that combines an effective diagnostic tool with an impactful management algorithm to improve the trajectory of the patient. Impedance monitoring has proven to be no different. IN-TIME was a randomized, controlled, multicenter trial of 716 patients with chronic heart failure and NYHA class II or III symptoms with a dual chamber implantable cardioverter-defibrillator (ICD) or cardiac resynchronization therapy-defibrillator (CRT-D) [19]. The primary end point was a composite score of all-cause death, HFH, change in NYHA class, and change in patient global self-assessment. The intervention arm was significantly better and this was overall driven by an improvement in the mortality rate (Fig. 3). However, multiple other studies using impedance fluid alerts have not shown benefit in clinical outcomes, such as OptiLink-HF, MORECARE, and REM-HF [32-34]. The Diagnostic Outcome Trial in Heart Failure (DOT-HF) study showed that measurement of intrathoracic impedance and impedancederived fluid index using ICD or CRT with an audible patient alert did not improve outcome and counterintuitively increased heart failure hospitalizations and outpatient visits in heart failure patients [35]. One explanation for the negative findings of this study relates to the lack of impedance-based monitoring treatment algorithms. Since impedance measurements provide only a relative rather than an absolute measurement of lung fluid content, the appropriate clinical response to a change in the impedance value is unclear. In DOT-HF, it appeared that alerting patients and clinicians without appropriate clear clinical treatment response caused increased concern and a paradoxical increased likelihood of hospital admission.

CIED impedance has some important limitations. Isolated cor pulmonale may be less well appreciated if presenting with predominantly right-sided signs and symptoms, without changes in intrathoracic fluid volume. Real-world experience with these devices dictates they are often followed by an electrophysiology or device clinic. Unless appropriate infrastructure is implemented, the clinic or team following the patient's device measurements may be separate from the team managing the patient's heart failure. The device data is also not immediately available to the patient, missing out on an opportunity to generate a patient's interest in their own heart failure management. In addition, factors such as obstructive lung disease and pulmonary lymphatic drainage can alter the accurate assessment of intrathoracic impedance. 

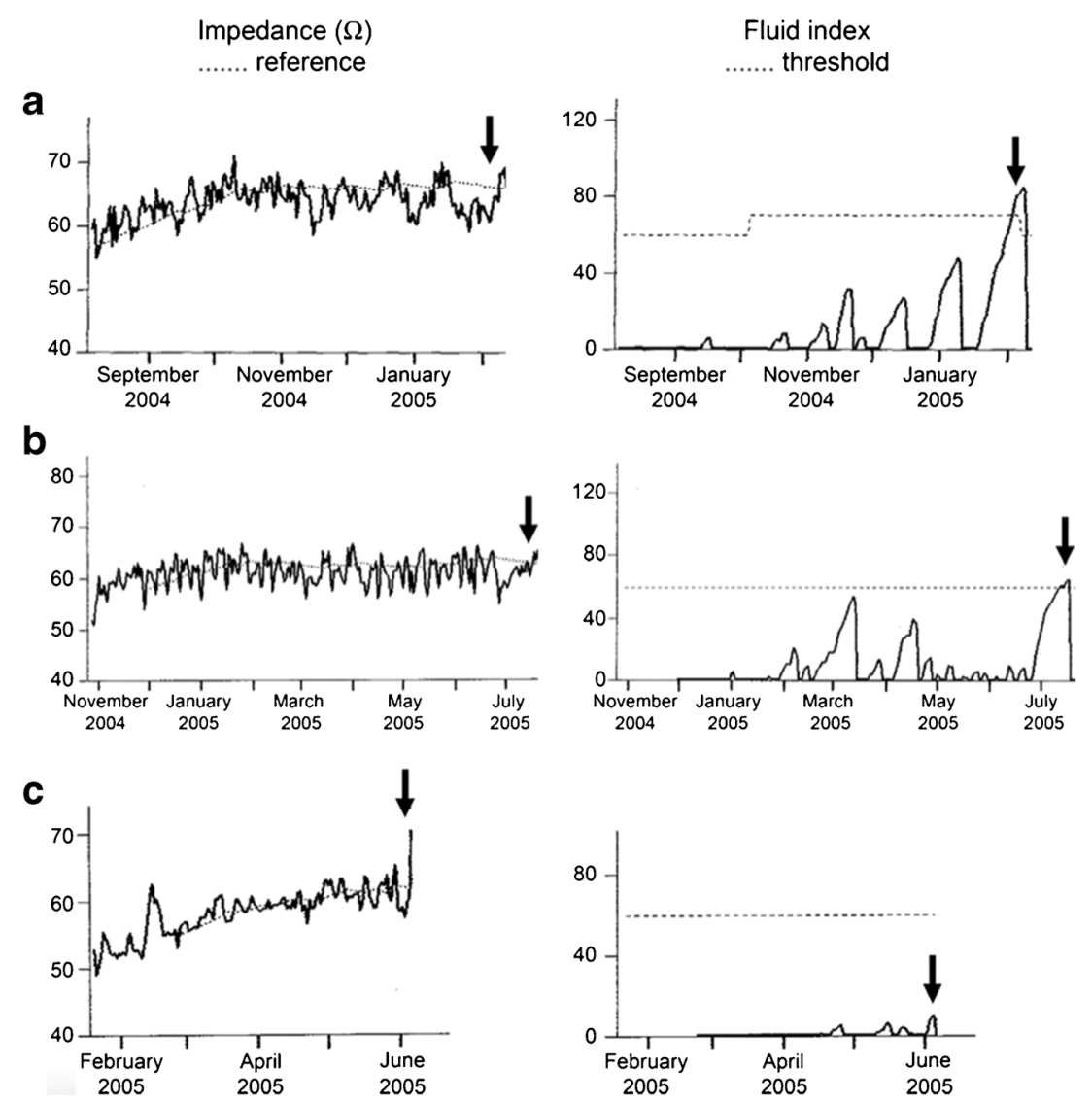

Fig. 2 Examples of different alert events. a True-positive alert in a 39year-old female who received the device because of DCM, EF of $20 \%$, complete heart block, and NYHA class III symptoms. In February 2005, an unscheduled follow-up was triggered by an alert that had first sounded the previous day. The patient denied worsening of HF symptoms, but admitted incompliance with regard to fluid intake in the last weeks. Her body weight had increased by $4 \mathrm{~kg}$ since the preceding visit, and chest Xray revealed signs of acute pulmonary congestion. Diuretic doses were increased and fluid intake was restricted. Six days later, her body weight had normalized and daily impedance was above the reference. Note that transiently the fluid index threshold had been programmed empirically from 60 to 70 in this patient. b False-positive alert in a 59-year-old male who received the device because of DCM, EF of $15 \%$, LBBB, and NYHA class III symptoms. In July 2005, the alert triggered an unscheduled follow-up $(\downarrow)$ when no evidence of HF deterioration was

\section{Wireless Hemodynamic Monitoring}

A fundamental shift in remote monitoring began with the advent of devices that directly and wirelessly measured intracardiac hemodynamics. Ambulatory pulmonary artery (PA) monitoring as a concept is far from new, with initial studies in humans dating back to the early 1980s [36]. Since then, there have been successive iterations of PA monitors, typically small feasibility studies [37-40]. The Chronicle device was comprised of a pectoral area memory device and transvenous electrode containing a pressure sensor which was deployed in the right ventricular outflow tract. A 2003 study of this device showed a clear signal of increased PA pressures at least 4 days prior to HFH [41]. This is a fundamental difference in

found. Note that daily impedance had increased, returning close to the reference at the time of follow-up visit. $\mathbf{c}$ False-negative alert in a 57-yearold male with ICM, EF of $20 \%$, LBBB, and NYHA class II symptoms at the time the resynchronization device was exchanged because of battery depletion. In June 2005, the patient was hospitalized for global HF decompensation $(\downarrow)$. Device interrogation revealed that daily impedance had decreased below the reference, but the accumulated difference between daily impedance and reference impedance (fluid index) was not sufficient to trigger the alert. Note the rapid increase in intrathoracic impedance during hospitalization and intensified diuretic therapy. DCM, dilative cardiomyopathy; EF, LV ejection fraction; ICM, ischaemic cardiomyopathy; LBBB, complete left bundle branch block. With permission from Oxford University Press-Journals, original figure from Vollmann et al. European Heart Journal (2007) 28, 1835-1840

hemodynamic data as compared to more generalized data such as weight or blood pressure. The pressure rises often several days prior to an event, and during this time, weight may remain unchanged.

While promising, the Chronicle device was evaluated in the Compass-HF trial and did not meet its primary endpoint. It was a single-blinded, prospective, multicenter study of 274 patients with HFpEF or HFrEF and NYHA class III or IV symptoms. The primary endpoint of heart failure events was $21 \%$ lower in the intervention cohort but this was not statistically significant $(=0.33)$ [42]. Evaluation of the trial suggests it was underpowered, the usual care arm did better than expected, and the guidance for how to manage changes in device-derived pressures was not optimal. 
Fig. 3 Kaplan-Meier curve as reported in IN-TIME study, 1year all-cause mortality in the telemonitoring group was 3.4 versus $8.7 \%$ in the control group (log-rank $p=0.004$; HR 0.36, 95\% CI 0.17-0.74), With permission from Elsevier, original figure from Hindricks et al. Lancet 2014; 384: 583-90

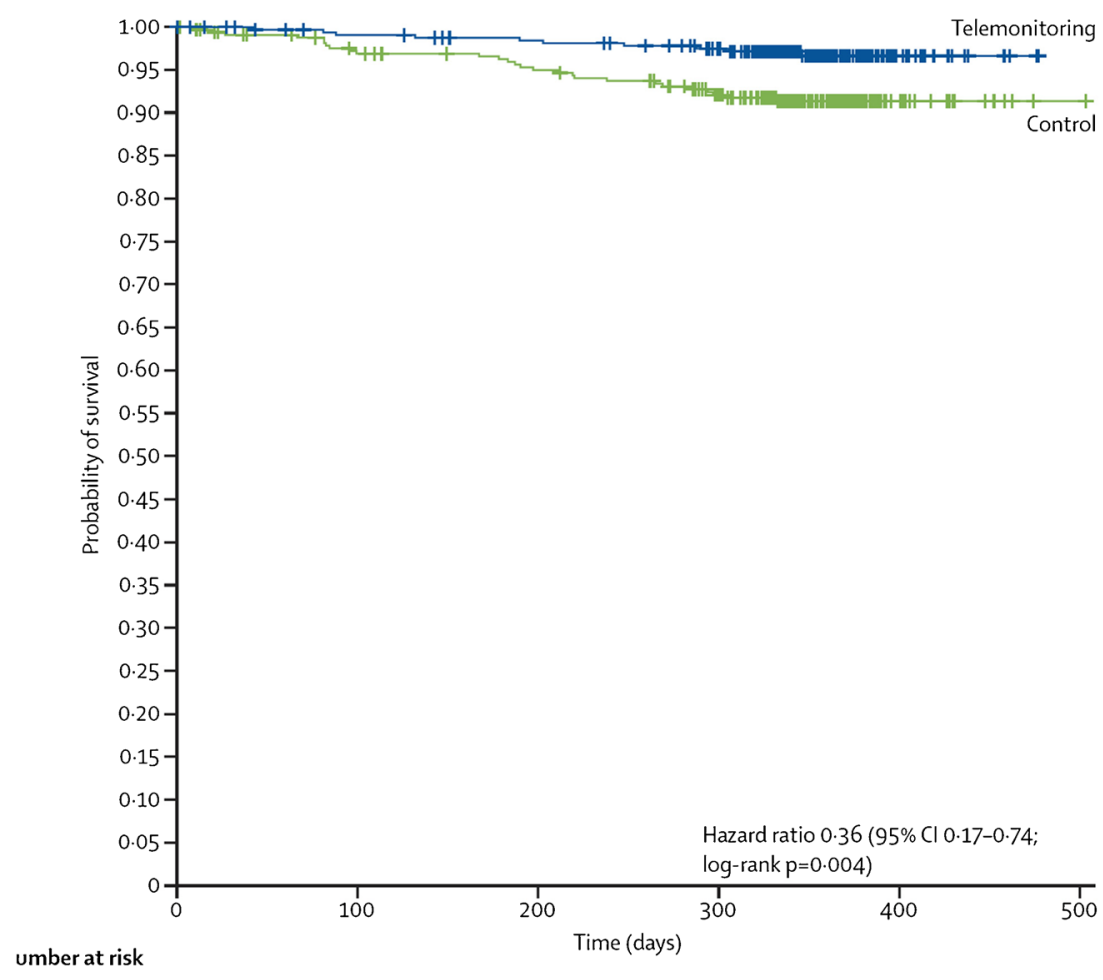

Another device was created which acted as a left atrial pressure sensor. The LAPTOP-HF trial was a prospective, multicenter, randomized controlled trial with HFH in the preceding 12 months and NYHA class III symptoms [43]. This device was implanted via trans-septal puncture to position the lead tip (sensor) into the left atrium. Patients received $150 \mathrm{mg}$ aspirin daily and at least 6 months of clopidogrel $75 \mathrm{mg}$ daily, and measured pressures at home twice daily using a handheld device. The study enrollment was stopped prematurely due to complications involving the trans-septal puncture. However, a follow-up study of those patients in the intervention arm who did complete the trial had a $41 \%$ reduction in $\mathrm{HFH}$ at 1 year [44].

The Cardiac Microelectromechanical system (CardioMEMSTM) is a passive wireless pressure sensor of pulmonary artery pressure. CardioMEMS ${ }^{\mathrm{TM}}$, first introduced in 2006, consists of an implantable pressure sensor, an external communication antenna, and an intravenous delivery system to deploy the sensor in the pulmonary artery. The sensor design is simple; it consists of membrane and a sealed cavity. The membrane responds to and deflects under pressure. Within the sealed cavity, a coil and capacitor transduce the membrane deflection into an electrical signal. The pressure sensor capitalizes on microelectromechanical system technology that can scale these deformable membranes into a small form that measures $3.5 \times 15 \times 2 \mathrm{~mm}$, suitable for endovascular delivery and permanent implantation [45]. The sensors are interrogated with an external communication antenna which is held against the patient's side or back in the approximate area of deployment of the sensor and a real-time waveform of the pressure environment is extracted. This device is battery free and the external antenna provides power to the implanted device by inductive coupling, that is when change in current through one wire (the external antenna) induces a voltage across the ends of the other wire (pressure sensor) through electromagnetic induction [46].

The system is calibrated for atmospheric pressure using a built-in sensor on the external antenna. Calibration is done at the time of device implantation with Swan-Ganz method for pulmonary artery pressure measurement as reference for calibration. On each scheduled visit, pulmonary artery pressure should be noninvasively determined using the external measurement antenna [47].

The wireless CardioMEMS ${ }^{\mathrm{TM}}$ pulmonary artery monitoring device was approved by the US Food and Drug Administration (FDA) in 2014 to monitor pulmonary artery pressure and heart rate in heart failure patients (heart failure with preserved ejection fraction or reduced ejection fraction) with NYHA class III symptoms who have been hospitalized during the previous year. The pressure sensor has a small size, good safety profile, simple implantation procedure, and provides an accurate pulmonary artery pressure measurement comparable to the Swan Ganz method (Fig. 4) [47, 48].

FDA approval was based on the CardioMEMS ${ }^{\text {тм }}$ Heart Sensor Allows Monitoring of Pressure to Improve Outcomes in NYHA Class III Heart Failure Patients (CHAMPION) trial [48]. This multicenter randomized controlled trial enrolled patients with NYHA class III symptoms with any LVEF and a HFH in the prior year and implanted device in the intervention arm. The primary endpoint was rate of HFH at 6 months. 

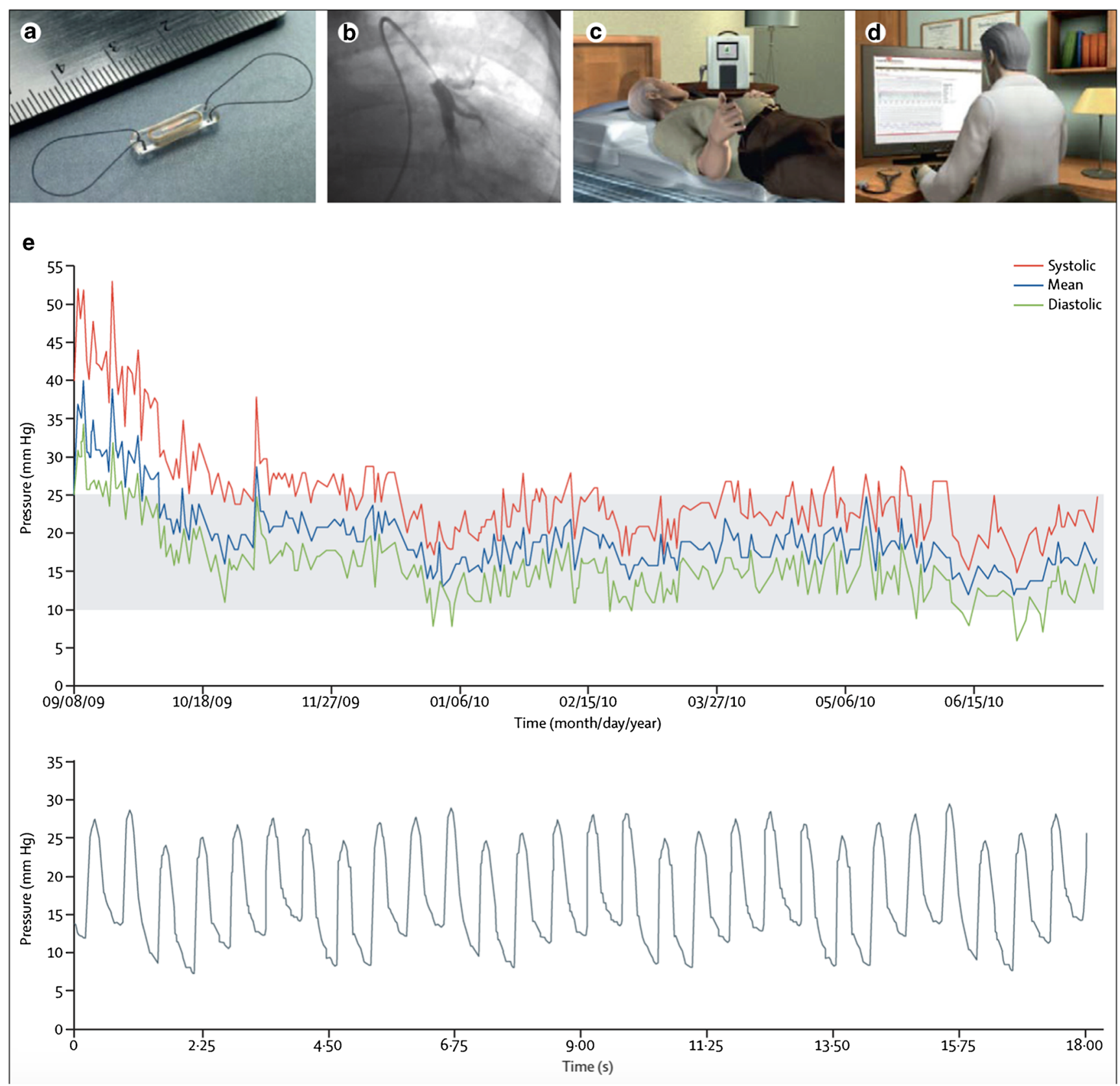

Fig. 4 Cardio-MEMS ${ }^{\mathrm{TM}}$ monitoring system. a CardioMEMS sensor. b Transcatheter is implanted into a distal branch of the left pulmonary artery. c Patient is instructed to take daily pressure readings from home using the home electronics. d Information transmitted from the monitoring system to the database is immediately available to the

Patients were considered hypervolemic when pulmonary artery systolic pressure, pulmonary artery diastolic pressure, or mean pulmonary artery were above the range of 15 to $35 \mathrm{mmHg}, 8$ to $20 \mathrm{mmHg}$, and 10 to $25 \mathrm{mmHg}$ respectively. Treatment involved initiation or intensification of diuretic agents, long-acting nitrates, and/or education regarding dietary salt and fluid restrictions. CardioMEMSTM guided therapy led to a $37 \%$ reduction in heart failure hospitalizations at 1 year [48]. The extended efficacy of pressure-directed heart clinical team for review. e Transmitted information consists of pressure trend information and individual pulmonary artery pressure waveforms. With permission from Elsevier, original figure from Abraham et al. Lancet 2011 Feb 19;377(9766):658-66

failure therapy was demonstrated using CHAMPION trial data. Heart failure hospitalizations were reduced by $33 \%$ over the randomized access process and $48 \%$ over the open access process over 31 months follow-up (Fig. 5) [49]. Reductions in HFH were observed regardless of reduced or preserved ejection fraction [50]. An observational study also showed that patients with CardioMEMS ${ }^{\mathrm{TM}}$ had an even more pronounced improvement in PA pressures over time as compared to CHAMPION data [51]. 


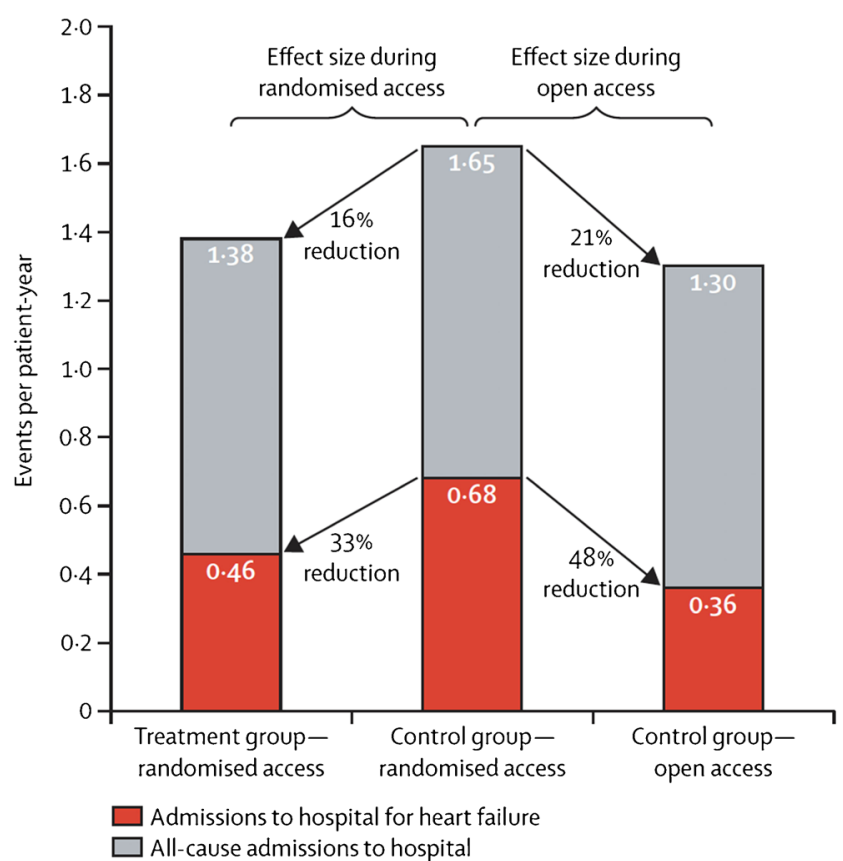

Fig. 5 Effect of pulmonary artery pressure-guided heart failure management on rates of admission to hospital. Treatment effect sizes in favor of pulmonary artery pressure-guided management observed during open access were similar to those observed during randomized access. With permission from the American Heart Association, original figure from Abraham et al. Lancet 2016; 387: 453-61

Long-term safety is excellent with no sensor failures reported. During the first 6 months of the CHAMPION trial, 0.02 events per patient-year device-related or system-related complications were reported. CardioMEMS ${ }^{\mathrm{TM}}$ safety profile compares favorably to that of right heart catheterization [49]. In heart failure patients with comorbid COPD, CardioMEMSTM directed heart failure management showed a reduction in respiratory hospitalizations due to pneumonia and COPD exacerbations [52]. One possible explanation is avoidance of volume overload by using pressure-guided therapy can decrease risk for pulmonary vascular stress, thus also preventing respiratory hospitalizations in addition to HFHs.

The CardioMEMS ${ }^{\mathrm{TM}} \mathrm{HF}$ system has been shown to have other benefits and roles as well. Rathman and colleagues reported that patients relayed improvements in perception of disease control and symptom reduction, while being satisfied with use of the system [53]. A small pilot study in left ventricular assist device (LVAD) patients demonstrated that none of the patients who received a CardioMEMS ${ }^{\mathrm{TM}}$ device and associated optimization before LVAD surgery had the occurrence of the primary endpoint of mortality, right-sided HF, or renal replacement therapy [54]. Another population of interest is pulmonary hypertension (PH). A 2015 study by Benza and colleagues retrospectively evaluated the CHAMPION trial patients and identified those with WHO Group II PH, about 59\% of the cohort [55]. As compared to non-PH patients, $\mathrm{PH}$ patients had higher mortality and HFH rates. PH patients in the CardioMEMS ${ }^{\text {TM }}$ arm had a significant reduction in $\mathrm{HFH}$ rates. Expanding to the diagnosis of pulmonary arterial hypertension (PAH), a small study of 26 patients with $\mathrm{PAH}$ and NYHA class III or IV symptoms had CardioMEMS ${ }^{\mathrm{TM}}$ implantations. The device was found to be safe and feasible to use in this population. Patients experienced a reduction in PA pressures, an increase in cardiac output, and an improvement in NYHA class and quality of life assessments [56].

There are a few limitations to the CardioMEMSTM HF system. Systolic pulmonary artery pressure, as determined with CardioMEMS $^{\mathrm{TM}}$, tends to be higher than Swan Ganz measurements. This is because the systolic parameter is derived from the highest frequency part of the waveform, the most sensitive parameter to differences in measurement method and sampling rate [47]. While it is FDA approved to reduce $\mathrm{HFH}$, its use has not been validated in other heart failure states such as congenital heart disease, isolated right heart failure, and patients with advanced kidney disease or requirement of renal replacement therapy. There are some contraindications to device placement, such as chest circumference $>165 \mathrm{~cm}$, history of recurrent pulmonary embolism, mechanical tricuspid or pulmonic valve replacements, or any contraindication to right heart catheterization [48]. Interference may come from household items such as an electric blanket or metal near the antenna. As new intracardiac devices are FDA approved, such as the new cardiac contractility modulator, it is not clear if they will lead to interference. CardioMEMS ${ }^{\mathrm{TM}}$ is also only approved for NYHA III patients, although the upcoming GUIDE-HF trial is set to answer this issue as it included NYHA classes II and IV [57].

Additional data supporting the effectiveness of the CardioMEMS HF System has been published. The 1-year results of the CardioMEMSTM Post-Approval Study, a multicenter, prospective, open-label, observational, single-arm trial of 1200 patients enrolled across 104 US sites, demonstrated a $57 \%$ reduction in $\mathrm{HFH}$ at 1 year compared with the year before device implantation [58]. Adherence rate to daily pressure transmission was $76 \pm 24 \%$ and PAP declined significantly. The rate of all-cause hospitalization was also significantly reduced. The results were consistent across multiple predefined subgroups, including by LVEF subgroup. Similarly, the CardioMEMS European Monitoring Study for Heart Failure (MEMS-HF) demonstrated a significant $62 \%$ reduction in the rate of HF hospitalization, comparing the 12 months post- versus pre-implant [59].

Finally, there have been mixed conclusions on the cost/benefit calculations for the CardioMEMSTM system. Sandhu and colleagues created a model that suggested a cost of $\$ 71,462$ per quality-adjusted life year (QALY) gained for a CardioMEMSTM-implanted patient [60]. An accompanying editorial suggested this is in the intermediate-value zone, and even this was questioned by the authors given the singular 
trial, single-blinding, and the impact of deviations from protocol approved management algorithms [61]. They also pointed out that if the trend toward mortality benefit is not seen in real-world data, the cost is closer to $\$ 150,000$ per QALY. This was further challenged by three subsequent studies that suggested better cost-effectiveness bordering on intermediate to high value [62-64].

\section{COVID-19 Pandemic}

A new stressor for heart failure patients is the COVID-19 pandemic. This highly contagious and morbid viral illness has fundamentally altered the preferred setting of the physician-patient interaction. Of 3080 patients with COVID19 in the CARD-COVID study, $4.9 \%$ had a history of HF, were found to be more likely to develop acute heart failure, and had a higher mortality over follow-up [65]. While most heart failure physicians base their management on a complete in-person history and physical exam, the sharp rise in utilization of telemedicine makes this assessment challenging or even impossible. It is this situation that highlights the value of remote monitoring, allowing the patient to be appropriately evaluated in the safety of their own home. The Canadian Heart Failure Society recommended adoption of remote monitoring and especially wireless hemodynamic devices as the most promising to augment the virtual heart failure clinic [66].

\section{Future Directions}

Technology is becoming ever woven into our lives, collecting biometric data as easily as telling the time. To this point, even smart watches are now giving a wide variety of physiologic information, including the presence of atrial fibrillation in the Apple Heart Study [67]. The era of personalized medicine has already begun, but it is still challenging to separate a "signal" from "noise." There is a clear space for application of machine learning and artificial intelligence to leverage computing power, allowing recognition of patterns in the sea of data and improvement on existing treatment algorithms. This may also improve clinical trial design to demonstrate clear benefit to outcomes with these monitoring devices. Further efforts to miniaturize devices and seamlessly integrate hemodynamic assessment into everyday life can provide robust information to keep heart failure patients compensated.

Despite the outcomes of the previous left atrial pressure sensor in the LAPTOP trial, there is a new left atrial pressure sensor in early preclinical testing. The V-LAP device is a left atrial leadless and wireless sensor that is implanted in the septum. The V-LAP sensor records an atrial ECG and provides left atrial waveform morphology, potentially allowing other comorbidities such as valvular regurgitation and arrhythmias to be detected. This is currently being evaluated in the VECTOR-HF trial in Europe [68]. Another device not yet commercially approved is a different type of pulmonary artery pressure sensor called Cordella from Endotronix, Inc. It uses similar technology (microelectromechanical) but the device is implanted in the right pulmonary artery, and patients use a handheld device in an upright seated position to measure pressures. A larger clinical trial, PROACTIVE-HF, is ongoing after the initial SIRONA trial showed the device implantation to be safe and readings to be accurate [69].

\section{Conclusions}

Remote monitoring and specifically wireless hemodynamic monitoring are valuable tools that can assist heart failure clinics in keeping patients optimized, out of the hospital and with sustained quality of life. Trial data has been mixed highlighting the importance of tying the data from these devices to an effective and preemptive management strategy. As cardiac medical devices become smaller, smarter, and more automated in their data collection, remote monitoring will continue to evolve into an indispensable part of the heart failure patient evaluation, especially with an emerging emphasis on virtual visits in the era of COVID-19.

\section{Compliance with Ethical Standards}

Conflict of Interest Matthew Lander and Nael Aldweib have no conflict of interest.

William T. Abraham reports personal fees from Abbott, during the conduct of the study, and during the last 36 months, he received consulting fees from Boehringer Ingelheim, CVRx, Edwards Lifesciences, Impulse Dynamics, and Respicardia, salary support from V-Wave Medical, and research support from the U.S. National Institutes of Health National Heart, Lung, and Blood Institute, all for studies performed within the heart failure arena.

Human and Animal Rights and Informed Consent All reported studies/ experiments with human or animal subjects performed by the authors have been previously published and complied with all applicable ethical standards (including the Helsinki declaration and its amendments, institutional/national research committee standards, and international/national/institutional guidelines).

\section{References}

Papers of particular interest, published recently, have been highlighted as:

- Of importance

•. Of major importance

1. Hunt SA, Abraham WT, Chin MH, Feldman AM, Francis GS, Ganiats TG, et al. 2009 focused update incorporated into the ACC/AHA 2005 Guidelines for the Diagnosis and Management 
of Heart Failure in Adults. Circulation. 2009;119(14):e391-479. https://doi.org/10.1161/CIRCULATIONAHA.109.192065.

2. Barker WH, Mullooly JP, Getchell W. Changing incidence and survival for heart failure in a well-defined older population, 19701974 and 1990-1994. Circulation. 2006;113(6):799-805. https:// doi.org/10.1161/CIRCULATIONAHA.104.492033.

3. Heidenreich PA, Albert NM, Allen LA, Bluemke DA, Butler J, Fonarow GC, et al. Forecasting the impact of heart failure in the United States: a policy statement from the American Heart Association. Circ Heart Fail. 2013;6(3):606-19. https://doi.org/10. 1161/HHF.0b013e318291329a.

4. Chang PP, Chambless LE, Shahar E, Bertoni AG, Russell SD, Ni $\mathrm{H}$, et al. Incidence and survival of hospitalized acute decompensated heart failure in four US communities (from the Atherosclerosis Risk in Communities Study). Am J Cardiol. 2014;113(3):504-10. https://doi.org/10.1016/j.amjcard.2013.10.032.

5. Dunlay SM, Redfield MM, Weston SA, Therneau TM, Hall Long $\mathrm{K}$, Shah ND, et al. Hospitalizations after heart failure diagnosis. J Am Coll Cardiol. 2009;54(18):1695-702. https://doi.org/10.1016/j. jacc.2009.08.019.

6. Bello NA, Claggett B, Desai AS, McMurray JJ, Granger CB, Yusuf $\mathrm{S}$, et al. Influence of previous heart failure hospitalization on cardiovascular events in patients with reduced and preserved ejection fraction. Circ Heart Fail. 2014;7(4):590-5. https://doi.org/10.1161/ CIRCHEARTFAILURE.113.001281.

7. Chartbook. https://www.cmshospitalchartbook.com/ (2020). Accessed.

8. Steinberg BA, Zhao X, Heidenreich PA, Peterson ED, Bhatt DL, Cannon CP, et al. Trends in patients hospitalized with heart failure and preserved left ventricular ejection fraction: prevalence, therapies, and outcomes. Circulation. 2012;126(1):65-75. https://doi. org/10.1161/CIRCULATIONAHA.111.080770.

9. Benjamin EJ, Virani SS, Callaway CW, Chamberlain AM, Chang AR, Cheng S, et al. Heart Disease and Stroke Statistics-2018 update: a report from the American Heart Association. Circulation. 2018;137(12):e67-e492. https://doi.org/10.1161/CIR. 0000000000000558 .

10. Greene SJ, Butler J, Albert NM, DeVore AD, Sharma PP, Duffy CI, et al. Medical therapy for heart failure with reduced ejection fraction. J Am Coll Cardiol. 2018;72(4):351-66. https://doi.org/10. 1016/j.jacc.2018.04.070.

11. Doukky R, Avery E, Mangla A, Collado FM, Ibrahim Z, Poulin $\mathrm{MF}$, et al. Impact of dietary sodium restriction on heart failure outcomes. JACC Heart Fail. 2016;4(1):24-35. https://doi.org/10. 1016/j.jchf.2015.08.007

12. <Chaudhry-2010-TELE HF trial.pdf>.

13. Krum H, Forbes A, Yallop J, Driscoll A, Croucher J, Chan B, et al. Telephone support to rural and remote patients with heart failure: the Chronic Heart Failure Assessment by Telephone (CHAT) study. Cardiovasc Ther. 2013;31(4):230-7. https://doi.org/10. 1111/1755-5922.12009.

14. <Gattis-1999-Pharm study.pdf>.

15. Ferrante D, Varini S, Macchia A, Soifer S, Badra R, Nul D, et al. Long-term results after a telephone intervention in chronic heart failure: DIAL (randomized trial of phone intervention in chronic heart failure) follow-up. J Am Coll Cardiol. 2010;56(5):372-8. https://doi.org/10.1016/j.jacc.2010.03.049.

16. Koehler F, Koehler K, Deckwart O, Prescher S, Wegscheider K, Kirwan B-A, et al. Efficacy of telemedical interventional management in patients with heart failure (TIM-HF2): a randomised, controlled, parallel-group, unmasked trial. Lancet. 2018;392(10152): 1047-57. https://doi.org/10.1016/s0140-6736(18)31880-4.

17. Ong MK, Romano PS, Edgington S, Aronow HU, Auerbach AD, Black JT, et al. Effectiveness of remote patient monitoring after discharge of hospitalized patients with heart failure: the better effectiveness after transition - heart failure (BEAT-HF) randomized clinical trial. JAMA Intern Med. 2016;176(3):310-8. https://doi. org/10.1001/jamainternmed.2015.7712.

18. Packer M. The neurohormonal hypothesis: a theory to explain the mechanism of disease progression in heart failure. J Am Coll Cardiol. 1992;20(1):248-54.

19. Hindricks G, Taborsky M, Glikson M, Heinrich U, Schumacher B, Katz A, et al. Implant-based multiparameter telemonitoring of patients with heart failure (IN-TIME): a randomised controlled trial. Lancet. 2014;384(9943):583-90. https://doi.org/10.1016/s01406736(14)61176-4.

20. Adamson PB, Smith AL, Abraham WT, Kleckner KJ, Stadler RW, Shih A, et al. Continuous autonomic assessment in patients with symptomatic heart failure: prognostic value of heart rate variability measured by an implanted cardiac resynchronization device. Circulation. 2004;110(16):2389-94. https://doi.org/10.1161/01. Cir.0000139841.42454.78.

21. Summers RL, Shoemaker WC, Peacock WF, Ander DS, Coleman TG. Bench to bedside: electrophysiologic and clinical principles of noninvasive hemodynamic monitoring using impedance cardiography. Acad Emerg Med. 2003;10(6):669-80. https://doi.org/10. 1111/j.1553-2712.2003.tb00054.x.

22. Pomerantz M, Delgado F, Eiseman BEN. Clinical evaluation of transthoracic electrical impedance as a guide to intrathoracic fluid volumes. Annals of Surgery. 1970;171(5).

23. Luepker RV, Michael JR, Warbasse JR. Transthoracic electrical impedance; quantitative evaluation of a non-invasive measure of thoracic fluid volume. Am Heart J. 1973;85(1):83-93. https://doi. org/10.1016/0002-8703(73)90529-2.

24. Yamamoto Y, Yamamoto T, Ozawa T. Characteristics of skin admittance for dry electrodes and the measurement of skin moisturisation. Med Biol Eng Comput. 1986;24(1):71-7. https:// doi.org/10.1007/bf02441608.

25. Yamamoto T, Yamamoto Y. Electrical properties of the epidermal stratum corneum. Med Biol Eng. 1976;14(2):151-8. https://doi.org/ $10.1007 / \mathrm{bf02478741.}$

26. Yu CM, Wang L, Chau E, Chan RH, Kong SL, Tang MO, et al. Intrathoracic impedance monitoring in patients with heart failure: correlation with fluid status and feasibility of early warning preceding hospitalization. Circulation. 2005;112(6):841-8. https://doi.org/ 10.1161/CIRCULATIONAHA.104.492207.

27. Wang L, Lahtinen S, Lentz L, Rakow N, Kaszas C, Ruetz L, et al. Feasibility of using an implantable system to measure thoracic congestion in an ambulatory chronic heart failure canine model. Pacing Clin Electrophysiol. 2005;28(5):404-11. https://doi.org/10.1111/j. 1540-8159.2005.40009.x.

28. Whellan DJ, Ousdigian KT, Al-Khatib SM, Pu W, Sarkar S, Porter $\mathrm{CB}$, et al. Combined heart failure device diagnostics identify patients at higher risk of subsequent heart failure hospitalizations: results from PARTNERS HF (Program to Access and Review Trending Information and Evaluate Correlation to Symptoms in Patients With Heart Failure) study. J Am Coll Cardiol. 2010;55(17):1803-10. https://doi.org/10.1016/j.jacc.2009.11.089.

29. Abraham WT, Compton S, Haas G, Foreman B, Canby RC, Fishel $\mathrm{R}$, et al. Intrathoracic impedance vs daily weight monitoring for predicting worsening heart failure events: results of the Fluid Accumulation Status Trial (FAST). Congest Heart Fail. 2011;17(2):51-5. https://doi.org/10.1111/j.1751-7133.2011. 00220.x.

30. Ahmed FZ, Taylor JK, Green C, Moore L, Goode A, Black P, et al. Triage-HF Plus: a novel device-based remote monitoring pathway to identify worsening heart failure. ESC Heart Fail. 2020;7(1):10716. https://doi.org/10.1002/ehf2.12529.

31. Boehmer JP, Hariharan R, Devecchi FG, Smith AL, Molon G, Capucci A, et al. A multisensor algorithm predicts heart failure events in patients with implanted devices: results from the 
MultiSENSE study. JACC Heart Fail. 2017;5(3):216-25. https:// doi.org/10.1016/j.jchf.2016.12.011.

32. Bohm M, Drexler H, Oswald H, Rybak K, Bosch R, Butter C, et al. Fluid status telemedicine alerts for heart failure: a randomized controlled trial. Eur Heart J. 2016;37(41):3154-63. https://doi.org/10. 1093/eurheartj/ehw099.

33. Boriani G, Da Costa A, Quesada A, Ricci RP, Favale S, Boscolo G, et al. Effects of remote monitoring on clinical outcomes and use of healthcare resources in heart failure patients with biventricular defibrillators: results of the MORE-CARE multicentre randomized controlled trial. Eur J Heart Fail. 2017;19(3):416-25. https://doi. org/10.1002/ejhf.626.

34. Morgan JM, Kitt S, Gill J, McComb JM, Ng GA, Raftery J, et al. Remote management of heart failure using implantable electronic devices. Eur Heart J. 2017;38(30):2352-60. https://doi.org/10. 1093/eurheartj/ehx227.

35. van Veldhuisen DJ, Braunschweig F, Conraads V, Ford I, Cowie $\mathrm{MR}$, Jondeau $\mathrm{G}$, et al. Intrathoracic impedance monitoring, audible patient alerts, and outcome in patients with heart failure. Circulation. 2011;124(16):1719-26. https://doi.org/10.1161/ circulationaha.111.043042.

36. Nathan AW, Perry SG, Cochrane T, Banim SO, Spurrell RA, Camm AJ. Ambulatory monitoring of pulmonary artery pressure. A preliminary clinical evaluation. Heart (British Cardiac Society). 1983;49(1):33-7. https://doi.org/10.1136/hrt.49.1.33.

37. <Simon-1992-A-new-system-for-ambulatory-pulmona.pdf $>$.

38. Steinhaus DM, Lemery R, Bresnahan DR Jr, Handlin L, Bennett T, Moore A, et al. Initial experience with an implantable hemodynamic monitor. Circulation. 1996;93(4):745-52. https://doi.org/10. 1161/01.cir.93.4.745.

39. Ohlsson A, Kubo SH, Steinhaus D, Connelly DT, Adler S, Bitkover $\mathrm{C}$, et al. Continuous ambulatory monitoring of absolute right ventricular pressure and mixed venous oxygen saturation in patients with heart failure using an implantable haemodynamic monitor: results of a 1 year multicentre feasibility study. Eur Heart J. 2001;22(11):942-54. https://doi.org/10.1053/euhj.2000.2419.

40. Magalski A, Adamson P, Gadler F, Boehm M, Steinhaus D, Reynolds $\mathrm{D}$, et al. Continuous ambulatory right heart pressure measurements with an implantable hemodynamic monitor: a multicenter, 12-month follow-up study of patients with chronic heart failure. J Card Fail. 2002;8(2):63-70. https://doi.org/10.1054/jcaf.2002. 32373 .

41. Adamson PB, Magalski A, Braunschweig F, Böhm M, Reynolds D, Steinhaus D, et al. Ongoing right ventricular hemodynamics in heart failure. J Am Coll Cardiol. 2003;41(4):565-71. https://doi. org/10.1016/s0735-1097(02)02896-6.

42. Bourge RC, Abraham WT, Adamson PB, Aaron MF, Aranda JM Jr, Magalski A, et al. Randomized controlled trial of an implantable continuous hemodynamic monitor in patients with advanced heart failure: the COMPASS-HF study. J Am Coll Cardiol. 2008;51(11): 1073-9. https://doi.org/10.1016/j.jacc.2007.10.061.

43. Ritzema J, Melton IC, Richards AM, Crozier IG, Frampton C, Doughty RN, et al. Direct left atrial pressure monitoring in ambulatory heart failure patients. Circulation. 2007;116(25):2952-9. https://doi.org/10.1161/circulationaha.107.702191.

44. Abraham WT, Adamson PB, Costanzo MR, Eigler N, Gold M, Klapholz M, et al. Hemodynamic monitoring in advanced heart failure: results from the LAPTOP-HF Trial. Journal of Cardiac Failure. 2016;22(11). https://doi.org/10.1016/j.cardfail.2016.09. 012.

45. Yu L, Kim BJ, Meng E. Chronically implanted pressure sensors: challenges and state of the field. Sensors (Basel). 2014;14(11): 20620-44. https://doi.org/10.3390/s141120620.

46. Allen MG. Micromachined endovascularly-implantable wireless aneurysm pressure sensors: from concept to clinic. The 13th International Conference on Solid-State Sensors, Actuators and
Microsystems, 2005 Digest of Technical Papers TRANSDUCERS '052005. p. 275-8 Vol. 1.

47. Verdejo HE, Castro PF, Concepcion R, Ferrada MA, Alfaro MA, Alcaino ME, et al. Comparison of a radiofrequency-based wireless pressure sensor to swan-ganz catheter and echocardiography for ambulatory assessment of pulmonary artery pressure in heart failure. J Am Coll Cardiol. 2007;50(25):2375-82. https://doi.org/10. 1016/j.jacc.2007.06.061.

48. Abraham WT, Adamson PB, Bourge RC, Aaron MF, Costanzo MR, Stevenson LW, et al. Wireless pulmonary artery haemodynamic monitoring in chronic heart failure: a randomised controlled trial. Lancet. 2011;377(9766):658-66. https://doi.org/10.1016/ S0140-6736(11)60101-3.

49. Abraham WT, Stevenson LW, Bourge RC, Lindenfeld JA, Bauman JG, Adamson PB. Sustained efficacy of pulmonary artery pressure to guide adjustment of chronic heart failure therapy: complete follow-up results from the CHAMPION randomised trial. Lancet. 2016;387(10017):453-61. https://doi.org/10.1016/s0140-6736(15) 00723-0.

50. Adamson PB, Abraham WT, Bourge RC, Costanzo MR, Hasan A, Yadav $\mathrm{C}$, et al. Wireless pulmonary artery pressure monitoring guides management to reduce decompensation in heart failure with preserved ejection fraction. Circ Heart Fail. 2014;7(6):935-44. https://doi.org/10.1161/CIRCHEARTFAILURE.113.001229.

51. Heywood JT, Jermyn R, Shavelle D, Abraham WT, Bhimaraj A, Bhatt K, et al. Impact of practice-based management of pulmonary artery pressures in 2000 patients implanted with the CardioMEMS sensor. Circulation. 2017;135(16):1509-17. https://doi.org/10. 1161/circulationaha.116.026184.

52. Krahnke JS, Abraham WT, Adamson PB, Bourge RC, Bauman J, Ginn $\mathrm{G}$, et al. Heart failure and respiratory hospitalizations are reduced in patients with heart failure and chronic obstructive pulmonary disease with the use of an implantable pulmonary artery pressure monitoring device. J Card Fail. 2015;21(3):240-9. https:// doi.org/10.1016/j.cardfail.2014.12.008.

53. Rathman LD, Sears S, Bourge RC, Desai AS, Heywood JT, Henderson J, et al. Patients report enhanced control over their heart failure during ambulatory hemodynamic monitoring. J Card Fail. 2020;26(10):S93-S4. https://doi.org/10.1016/j.cardfail.2020.09. 274.

54. Veenis JF, Brugts JJ. Remote monitoring of chronic heart failure patients: invasive versus non-invasive tools for optimising patient management. Neth Heart J. 2020;28(1):3-13. https://doi.org/10. 1007/s12471-019-01342-8.

55. Benza RL, Raina A, Abraham WT, Adamson PB, Lindenfeld J, Miller AB, et al. Pulmonary hypertension related to left heart disease: insight from a wireless implantable hemodynamic monitor. J Heart Lung Transplant. 2015;34(3):329-37. https://doi.org/10. 1016/j.healun.2014.04.014.

56. Benza RL, Gomberg-Maitland M, Elliott CG, Farber HW, Foreman AJ, Frost AE, et al. Predicting survival in patients with pulmonary arterial hypertension: the REVEAL risk score calculator 2.0 and comparison with ESC/ERS-based risk assessment strategies. Chest. 2019;156(2):323-37. https://doi.org/10.1016/j.chest.2019. 02.004 .

57. Lindenfeld J, Abraham WT, Maisel A, Zile M, Smart F, Costanzo MR, et al. Hemodynamic-GUIDEd management of Heart Failure (GUIDE-HF). Am Heart J. 2019;214:18-27. https://doi.org/10. 1016/j.ahj.2019.04.014.

58. Shavelle DM, Desai AS, Abraham WT, Bourge RC, Raval N, Rathman LD, et al. Lower rates of heart failure and all-cause hospitalizations during pulmonary artery pressure-guided therapy for ambulatory heart failure: one-year outcomes from the CardioMEMS Post-Approval Study. Circ Heart Fail. 2020;13(8): e006863. https://doi.org/10.1161/CIRCHEARTFAILURE.119. 006863 . 
59. Angermann CE, Assmus B, Anker SD, Asselbergs FW, Brachmann J, Brett ME, et al. Pulmonary artery pressure-guided therapy in ambulatory patients with symptomatic heart failure: the CardioMEMS European Monitoring Study for Heart Failure (MEMS-HF). Eur J Heart Fail. 2020;22:1891-901. https://doi. org/10.1002/ejhf.1943.

60. Sandhu AT, Goldhaber-Fiebert JD, Owens DK, Turakhia MP, Kaiser DW, Heidenreich PA. Cost-effectiveness of implantable pulmonary artery pressure monitoring in chronic heart failure. JACC Heart Fail. 2016;4(5):368-75. https://doi.org/10.1016/j. jchf.2015.12.015.

61. Dhruva SS, Krumholz HM. Championing effectiveness before cost-effectiveness. JACC Heart Fail. 2016;4(5):376-9. https://doi. org/10.1016/j.jchf.2016.02.001.

62. Cowie MR, Simon M, Klein L, Thokala P. The cost-effectiveness of real-time pulmonary artery pressure monitoring in heart failure patients: a European perspective. Eur J Heart Fail. 2017;19(5):6619. https://doi.org/10.1002/ejhf.747.

63. Martinson M, Bharmi R, Dalal N, Abraham WT, Adamson PB. Pulmonary artery pressure-guided heart failure management: US cost-effectiveness analyses using the results of the CHAMPION clinical trial. Eur J Heart Fail. 2017;19(5):652-60. https://doi.org/ 10.1002/ejhf.642.

64. Schmier JK, Ong KL, Fonarow GC. Cost-effectiveness of remote cardiac monitoring with the CardioMEMS heart failure system. Clin Cardiol. 2017;40(7):430-6. https://doi.org/10.1002/clc.22696.
65. Rey JR, Caro-Codon J, Rosillo SO, Iniesta AM, CastrejonCastrejon S, Marco-Clement I, et al. Heart failure in COVID-19 patients: prevalence, incidence and prognostic implications. Eur J Heart Fail. 2020. https://doi.org/10.1002/ejhf.1990.

66. Virani SA, Clarke B, Ducharme A, Ezekowitz JA, Heckman GA, McDonald $\mathrm{M}$, et al. Optimizing access to heart failure care in Canada during the COVID-19 pandemic. Can J Cardiol. 2020;36(7):1148-51. https://doi.org/10.1016/j.cjca.2020.05.009.

67. Perez MV, Mahaffey KW, Hedlin H, Rumsfeld JS, Garcia A, Ferris $\mathrm{T}$, et al. Large-scale assessment of a Smartwatch to identify atrial fibrillation. N Engl J Med. 2019;381(20):1909-17. https://doi.org/ 10.1056/NEJMoa1901183.

68. D'Amario D, Restivo A, Canonico F, Rodolico D, Mattia G, Francesco B, et al. Experience of remote cardiac care during the COVID-19 pandemic: the V-LAPTM device in advanced heart failure. Eur J Heart Fail. 2020;22(6):1050-2. https://doi.org/10.1002/ ejhf.1900.

69. Mullens W, Sharif F, Dupont M, Rothman AMK, Wijns W. Digital health care solution for proactive heart failure management with the Cordella Heart Failure System: results of the SIRONA first-inhuman study. Eur J Heart Fail. 2020;22:1912-9. https://doi.org/ 10.1002/ejhf.1870.

Publisher's Note Springer Nature remains neutral with regard to jurisdictional claims in published maps and institutional affiliations. 